\title{
Paraguay: Muchas nOVedades y POCO CAMbio
}

\section{Paraguay 2009: Many Differences and Little Change}

\author{
BRIAN TURNER* \\ Randolph-Macon College
}

\section{RESUMEN}

En un año sin elecciones y un nuevo gobierno histórico, 2009 parecía un año lleno de posibilidades de cambio importantes en las políticas del gobierno y en el proceso político. Sin embargo, las debilidades internas de la coalición que llevó a Fernando Lugo a la presidencia en 2008, los límites de las facultades reales del Poder Ejecutivo, y los grandes desafíos presentados por la estructura socioeconómica de Paraguay y por la herencia política de más de 60 años de gobierno de partido único hicieron de 2009 un año en el cual muy poco parecía cambiar.

Palabras clave: Paraguay, Ejecutivo, cambio.

\begin{abstract}
In a year without elections and an historic new government, 2009 seemed full of possibilities for important new directions in both policy and in the political process. However, the internal weaknesses of the coalition that brought Fernando Lugo to the presidency in 2008, the limits on the power of the executive branch, and the daunting challenges presented by Paraguay's socioeconomic structure and by the political inheritance of more than 60 years of single-party rule made 2009 a year in which very little seemed to change.
\end{abstract}

Key words: Paraguay, Executive, change. 
En un año sin elecciones generales o internas, mucho del enfoque de 2009 estuvo en los logros del nuevo gobierno histórico, que asumió el poder el 15 de agosto de 2008 con muchas expectativas después de haber derrotado al Partido Colorado que había gobernado el país desde 1947. El ex obispo Fernando Armindo Lugo Méndez, candidato de la Alianza Patriótica para el Cambio, y ahora Presidente de la República, cumplió sus primeros cien días en el gobierno en condiciones políticas bastante difíciles y sin una base fuerte que realzara su gobierno, pero con el triunfo histórico en las elecciones del 20 de abril de 2008 alentando las esperanzas del pueblo.

Sin embargo, los augurios no eran tan promisorios cuando minutos antes de la llegada del nuevo año una columna de subversivos vinculados con el Ejército del Pueblo Paraguayo (EPP) quemó un puesto militar en la zona de Tacuatí, Departamento de San Pedro. La falta de seguridad pública, marcada por el temor de que un foco guerrillero pudiera instalarse en el país y las acusaciones de la oposición política, arguyendo que figuras del Poder Ejecutivo apoyaban a los elementos del EPP, fueron algunos de los temas que el gobierno no pudo controlar. El año terminaría con el plagio del estanciero Fidel Zavala, el 15 de octubre, en el Departamento de Concepción, supuestamente por miembros del EPP. Zavala fue liberado el 17 de enero de 2010.

\section{LA COYUNTURA SOCIAL Y ECONÓMICA}

El manejo macroeconómico del 2009 mostró mejoras. El crecimiento económico del año fue proyectado por el Banco Central del Paraguay con una tasa del 5,56\%, manteniendo un razonable ritmo de crecimiento por tres años consecutivos, lo que marca grandes diferencias con años anteriores. La inflación en el 2009 fue de 1,9\%, la tasa más baja de los últimos 40 años (BCP, 2009). El índice de precios pagados al productor registró un fuerte crecimiento en comparación al del 2008, cuando los precios pagados al productor descendieron. El crecimiento fue especialmente fuerte en agricultura, con una tasa de 23,6\%. El Cuadro 1 sintetiza parte de estos resultados.

Cuadro 1: Indicadores de crecimiento (2002-2008)

\begin{tabular}{lccccccc}
\hline \multicolumn{1}{r}{ Indicadores de crecimiento } & 2002 & 2003 & 2004 & 2005 & 2006 & 2007 & 2008 \\
\hline Crecimiento del PBI (\%) & 0 & 4 & 4 & 4 & 4 & 7 & 6 \\
Crecimiento del PBI per cápita (\%) & -2 & 2 & 2 & 1 & 2 & 5 & 4 \\
\hline
\end{tabular}

Fuente: Banco Mundial.

Las importaciones y exportaciones descendieron de los índices más altos registrados en 2008. En 2009 el valor total de exportaciones fue de US\$ 3.167.000, una baja de $29 \%$ en comparación a 2008. El 53,8\% del descenso se puede atribuir al declive en las exportaciones de soja, el rubro más importante que contribuye con el $25 \%$ del valor total de exportaciones. El valor de exportaciones de la soja alcanzó su cúspide en 2008, 
registrando US\$ 1.485.311.000, mientras que en 2009 sólo ganó US\$ 787.159.000, un descenso del $47 \%$. El otrora producto de exportación por excelencia, el algodón, que en 1995 constituía el 29\% del valor total de exportaciones, continuó su desaparición de la economía paraguaya, registrando exportaciones que ahora sólo alcanzan el 1\% del valor total de exportaciones. El auge de la soja y la desaparición del algodón han tenido un fuerte impacto en las comunidades campesinas, en un proceso ya muy comentado desde que la soja superó definitivamente al algodón como el producto más rentable para el país, en 1996 (Fogel y Riquelme, 2005). Las importaciones tuvieron un valor total de US\$ 6.496.900, también una baja del 23,6\% en comparación al año 2008. El saldo de la balanza comercial en 2009 fue un déficit de US\$ 3.329.900 (BCP, 2010).

Paraguay es un país cuyas cultura y economía están fuertemente arraigadas en las zonas rurales y en el campesinado, pero en las últimas dos décadas se ha convertido en un país de población mayormente urbana, con la producción agrícola dominada por productores grandes. El Censo Agropecuario Nacional de 2008 muestra que desde 1991 ha disminuido el número de productores pequeños (con menos de 100 hectáreas de tierra) a 7,8\%. En 1991 los productores pequeños representaban el 95,9\% del total de productores aunque controlaban sólo el 11,9\% de las tierras. En 2008, los productores pequeños representaban el 93,8\% y controlaban ya sólo el 7,8\% de las tierras. En este tiempo la superficie cultivada se expandió en un 30,5\%. El tamaño medio (por valor medio, no por promedio) de fincas en 1991 era de 77,5 hectáreas, y en 2008 alcanzó 107,3 hectáreas. La concentración de tierras entre los productores también disminuyó. El tamaño promedio de las fincas más grandes de 10.000 hectáreas disminuyó en $23,9 \%$, pero esto no significa la reducción en la concentración de tierras en el sentido global. ${ }^{1}$

La tasa de desempleo y subocupación alcanzó 32,2\% en 2008, casi igual a las tasas registradas para 2005 y 2007 (DGEEC, 2008a). El desempleo abierto fue de 5,7\%, y la subocupación, visible y no visible, fue de $26,5 \%$.

La tasa de pobreza en el país bajó desde 2007 de 41,2\% a 37,9\% (DGEEC, 2008b). ${ }^{2}$ La pobreza urbana en 2007 fue medida en $33,6 \%$ y en 2008 en $30,2 \%$. También bajó la pobreza rural, de $51,8 \%$ en 2007 a $48,8 \%$ en 2008 . La pobreza extrema fue medida en $19 \%$ para 2008 .

El coeficiente Gini, que mide la distribución de riqueza, en el cual 0 indica una distribución igualitaria y 100 indica una concentración de riqueza perfecta, también muestra una tendencia de mejora, pero Paraguay, como otros países de la región, está entre los menos igualitarios del mundo.

Cálculos hechos por el autor de datos publicados en ABC Digital, 20 agosto 2009.

Dirección General de Estadística, Encuestas y Censos, “Mejora de la metodología de medición de pobreza en Paraguay. Resultados 1997-2008". Nota que la medición de pobreza ha cambiado. Antes se informó que la tasa de pobreza en 2007 fue de $35,6 \%$. 
Cuadro 2: Distribución de riqueza en el Paraguay

\begin{tabular}{rccr}
\hline & 2002 & 2005 & 2007 \\
\hline Coeficiente Gini & 58 & 54 & 53
\end{tabular}

Fuente: Banco Mundial.

Aunque varios voceros de los movimientos sociales y políticos, que apoyaran al Presidente Lugo, hablaban de vez en cuando de la posibilidad de defender al presidente "en las calles", si había una quiebra institucional, el público no le daba su entera confianza a la clase política. En una serie de publicaciones de Latin American Public Opinion Project de Vanderbilt University, el pueblo paraguayo aparece entre uno de los más desconfiados en su sistema político en América Latina. En el Cuadro 3 se pueden apreciar los promedios de 0 a 100, calculados en base a las respuestas de preguntas medidas por una escala Likert.

Cuadro 3: Opinión pública en el Paraguay, 2008

\begin{tabular}{lcl}
\hline \multicolumn{1}{c}{ Pregunta } & $\begin{array}{c}\text { Promedio } \\
\text { en Paraguay }\end{array}$ & \multicolumn{1}{c}{ Nota } \\
\hline $\begin{array}{l}\text { ¿Los partidos políticos escuchan a la } \\
\text { gente? (Corral, 2009) }\end{array}$ & 21,9 & Más bajo en la región \\
$\begin{array}{l}\text { ¿Hasta qué punto tiene confianza usted } \\
\text { en el Tribunal Supremo Electoral? } \\
\text { (Montalvo, 2009a) }\end{array}$ & 21,9 & $\begin{array}{l}\text { Más bajo en la región (seguido por Haití } \\
\text { con un promedio de 34,8) }\end{array}$ \\
$\begin{array}{l}\text { ¿Hasta qué punto tiene confianza usted } \\
\text { en las fuerzas armadas? (Montalvo, }\end{array}$ & 41,5 & $\begin{array}{l}\text { Segundo más bajo en la región (Argentina } \\
\text { figura con el promedio más bajo) }\end{array}$ \\
$\begin{array}{l}\text { 2009b) } \\
\begin{array}{l}\text { ¿Las autoridades deben respetar la ley } \\
\text { cuando luchan en contra del crimen? } \\
\text { (Cruz, 2009) }\end{array}\end{array}$ & 51,3 & $\begin{array}{l}\text { Entre los cuatro más bajos (Uruguay, } \\
\text { Honduras y Nicaragua figuran con } \\
\text { promedios más bajos) }\end{array}$ \\
$\begin{array}{l}\text { ¿El Presidente (debe) limitar la voz y } \\
\text { el voto de los partidos de la oposición? } \\
\text { (Orces, 2009a) }\end{array}$ & 23,6 & $\begin{array}{l}\text { Segundo más bajo en la región (Argentina } \\
\text { figura con el promedio más bajo) }\end{array}$ \\
$\begin{array}{l}\text { ¿El Presidente debe gobernar sin el } \\
\text { Congreso? (Orces, 2009b) }\end{array}$ & 23,0 & $\begin{array}{l}\text { Segundo más bajo en la región (Argentina } \\
\text { figura con el promedio más bajo) }\end{array}$ \\
\hline
\end{tabular}

En los Cuadros 4 y 5 se pueden apreciar las respuestas de los parlamentarios a varias preguntas en encuestas realizadas entre 2004-2008 por el Instituto de Iberoamérica de la Universidad de Salamanca, proyecto "Élites Parlamentarias Latinoamericanas" (Belmonte, 2009). 
Cuadro 4: Opinión de élites parlamentarias en el Paraguay: Desconfianza en instituciones

\begin{tabular}{lcl}
\hline \multicolumn{1}{c}{ Institución } & $\begin{array}{c}\text { \% poca o nada } \\
\text { confianza }\end{array}$ & \multicolumn{1}{c}{ Nota } \\
\hline Poder Judicial & 94,5 & Más alto nivel de desconfianza en la región \\
Partidos políticos & 44,6 & Total en América Latina, $48,4 \%$ \\
\hline
\end{tabular}

Cuadro 5: Opinión de élites parlamentarias en el Paraguay

\begin{tabular}{lcl}
\hline \multicolumn{1}{c}{ Pregunta } & $\begin{array}{c}\text { Porcentaje } \\
\text { afirmativo }\end{array}$ & Nota \\
\hline $\begin{array}{l}\text { Sin partidos no puede haber } \\
\text { democracia }\end{array}$ & 94,5 & $\begin{array}{l}\text { Segundo más alto en la región (después } \\
\text { de Costa Rica) }\end{array}$ \\
\hline
\end{tabular}

Los paraguayos y paraguayas muestran promedios bajos de confianza en instituciones importantes para la función de una democracia representativa. Aunque las élites parlamentarias paraguayas están entre las más insistentes en América Latina sobre la importancia de los partidos políticos (Cuadro 4), los ciudadanos no creen que los partidos escuchen a la gente y se registra en ellos mucha desconfianza. Y aunque los comicios generales más recientes no han sido muy viciados, la confianza en el Tribunal Superior de Justicia Electoral (TSJE) es muy baja. Los parlamentarios también desconfían de la TSJE y del Poder Judicial. 25\% de los parlamentarios contestaron a una encuesta en 2008 que tienen confianza en el TSJE. Es la tasa más baja en la región (Brenes, 2009).

Al contrario, las encuestas muestran que no hay mucho apoyo para el Poder Ejecutivo frente al Congreso. Esto se puede interpretar como oposición al mandatario del momento, y no tanto como apoyo al Congreso, o para mantener el balance entre poderes. En un análisis de los resultados de la Americas Barometer, 2006, los paraguayos respondieron con los niveles más bajos de actitud en el apoyo a la democracia liberal. Solo $42,7 \%$ de paraguayos mostraron estas actitudes, acompañados sólo por Haití (49\%) con menos de la mitad de encuestados (Carrión, 2008, 36).

\section{COYUNTURA POLÍTICA}

El Presidente Lugo comenzó el año en un contexto bastante complicado. Él ganó las elecciones de 2008 sin mayoría absoluta de votos (40,9\%). Su coalición (APC) incluyó a uno de los dos partidos tradicionales, el Partido Liberal Radical Auténtico (PLRA), que se sintió como la fuerza con el caudal más importante de votos pero con relaciones difíciles con el actual mandatario. Los partidos y movimientos más afines a las perspectivas del presidente habían logrado pocos votos, como se puede ver en la votación para el Senado, que se efectúa en listas cerradas y bloqueados en una sola 
circunscripción nacional. El PLRA casi empató con la Asociación Nacional RepublicanaPartido Colorado, el otro partido tradicional, obteniendo cada grupo un poco más del 27\% del voto para senadores. El PLRA ganó 14 escaños de los 45 en el Senado. El Partido País Solidario, la segunda fuerza electoral en la APC, ganó solo 3,25\% de los votos y un senador, al igual que el Partido Democrático Progresista y el Movimiento Popular Tekojojá. Así, con 17 senadores la APC no tenía mayoría. Tampoco la obtuvo en la Cámara de Diputados, donde el PLRA ganó 27 de los 80 escaños, y otros partidos de la Alianza sólo contribuyeron con 4 más.

Las relaciones entre el presidente y el PLRA siempre han sido complicadas por el fraccionalismo del partido, diferencias importantes en proyectos políticos, y los recelos personales que siempre han tenido una fuerte influencia en el proceso político paraguayo. El Vicepresidente, Federico Franco, presidente del PLRA con permiso, disputó con el Presidente Lugo políticas y nombramientos y los legisladores que respondían al vicepresidente no se pudieron contar como fieles a las iniciativas del Ejecutivo.

Otro aspecto difícil para Lugo fue el hecho de que después de 61 años en el poder los funcionarios públicos y los uniformados de las fuerzas armadas estaban muy "coloradizados". Los ministros nombrados por el presidente no esperaban tener el apoyo de los funcionarios, especialmente en las agencias con más empleados, pues los procesos de nombramiento resultaron muy politizados, como en el Ministerio de Educación y Cultura.

\section{PODERES DEL ESTADO}

\section{Poder Ejecutivo}

Como cualquier administración nueva, la conformación del gabinete fue un proceso de balance de fuerzas dentro de la coalición gobernante. Como se ha indicado, lo más destacado de este proceso, en agosto de 2008, fue la capacidad del Presidente Lugo de utilizar los nombramientos de liberales (PLRA) para aislar al grupo del vicepresidente Franco, también del PLRA.

En 2009 hubo cambios en seis carteras ministeriales, generando tensiones dentro del PLRA, y entre los miembros del gabinete. El conflicto se desató con la propuesta de darles un subsidio a los pequeños productores de sésamo ("sesameros"), castigados por la sequía, y quienes estaban mejor organizados en el Departamento de San Pedro, donde el presidente actuaba como obispo y el gobernador actual, José Ledesma, es importante aliado de Lugo. El sésamo es un rubro rentable que ha tomado el lugar del algodón en algunas zonas del país como producto campesino. La forma particular de la propuesta de subsidio parecía marginar al Ministerio de Agricultura y Ganadería (MAG), y en marzo casi renuncia el Ministro Cándido Vera Bejarano del PLRA. Sólo con la intervención del Vicepresidente Franco se evitó en ese momento la renuncia del ministro. Pero Vera ganó la ventaja en su conflicto interno con el Secretario General de la Presidencia, Miguel Ángel López Perito, y este último presentó su renuncia el 7 de abril. 
El 8 de abril, en plena Semana Santa, se desató el escándalo del año cuando Viviana Carrillo, una joven de 26 años, demandó a Fernando Lugo por la paternidad no asumida de su niño de dos años, Guillermo Armindo Carrillo Cañete. El lunes 13 de abril, Lugo reconoció la paternidad del niño, rechazó la renuncia de López Perito y anunció que habría cambios en el gobierno después del 20 de abril, primer aniversario de su histórica elección. En la fecha, Lugo destituyó a Vera Bejarano del MAG; a Horacio Galeano Perrone, Ministro de Educación y Cultura; a Martín Heisecke, Ministro de Industria y Comercio; y a Blas Llano, Ministro de Justicia y Trabajo. Los flamantes ministros, Enzo Cardozo, Luis Alberto Riart, Francisco Rivas Almada, y Humberto Blasco, respectivamente, son todos de los sectores del PLRA más adeptos a la línea del presidente.

Galeano Perrone es de trayectoria colorada y no representaba un grupo que apoyara políticamente al ejecutivo. Heisecke, del PLRA, tampoco tenía u perfil fuerte en su partido político. Blas Llano, senador de la República con permiso, se volvió a al Senado para fortalecer el bloque oficialista.

Al final del mes, Lugo nombró a Héctor Lacognata como Ministro de Relaciones Exteriores, en reemplazo de Alejandro Hamed. Lacognata estaba entre los fundadores del Partido Patria Querida, un partido centroderechista, pero en el mismo mes de abril renunció y se afilió con el Partido-Movimiento al Socialismo (P-MAS). Con este nombramiento, el gabinete está conformado por los colaboradores más leales de Lugo y empieza a verse el declive de la influencia del Vicepresidente Franco. López Perito y el Ministro del Interior Rafael Filizzola fueron los que salieron más fuertes de estos acontecimientos.

Cuadro 6: Conformación del Gabinete después del 28 de abril de 2009

\begin{tabular}{|c|c|c|}
\hline Ministerio & Ministro & Pertenencia partidaria \\
\hline $\begin{array}{l}\text { Secretario General y Jefatura } \\
\text { de Gabinete Civil }\end{array}$ & Miguel Ángel López Perito & Movimiento Popular Tekojojá \\
\hline Agricultura y Ganadería & Enzo Cardozo Jiménez & PLRA \\
\hline Defensa Nacional & Luis Bareiro Spaini & Militar (SR) \\
\hline Educación y Cultura & Luis Alberto Riart & PLRA \\
\hline Hacienda & Dionisio Borda & Independiente \\
\hline Industria y Comercio & Francisco Rivas Almada & PLRA \\
\hline Interior & Rafael Filizzola & Partido Democrático Progresista \\
\hline Justicia y Trabajo & Humberto Blasco & PLRA \\
\hline Relaciones Exteriores & Héctor Lacognata Zaragoza & $\begin{array}{l}\text { P-MAS, se separó del Partido Patria } \\
\text { Querida en abril de } 2009\end{array}$ \\
\hline Salud Pública y Bienestar Social & Esperanza Martínez & Movimiento Popular Tekojojá \\
\hline Obras Públicas y Comunicaciones & Efraín Alegre & PLRA \\
\hline $\begin{array}{l}\text { Secretaría de Información y } \\
\text { Comunicación para el } \\
\text { Desarrollo (SICOM) }\end{array}$ & Augusto Dos Santos & Movimiento Popular Tekojojá \\
\hline $\begin{array}{l}\text { Secretaría de Emergencia } \\
\text { Nacional (SEN) }\end{array}$ & Camilo Soares Machado & Partido-Movimiento al Socialismo \\
\hline
\end{tabular}


El 20 de abril, Benigna Leguizamón le interpuso una demanda a Lugo por la paternidad de su hijo Lucas Fernando, nacido en septiembre de 2002, cuando Lugo todavía era Obispo de San Pedro. Seguidamente, el 22 de abril, Damiana Hortensia Morán afirmó que Lugo era el padre de su hijo, nacido en diciembre de 2007. Lugo no ha reconocido a estos niños como suyos. Aunque el escándalo golpeó la imagen del presidente, políticamente él fue capaz de sobrevivir la crisis. En julio de 2009, la mitad (50,0\%) de las respuestas a una encuesta hecha por Angus Reid Global Monitor (2009) calificó la actuación del presidente como "buena" o "muy buena".

También se realizaron remociones en las fuerzas armadas. El 4 de noviembre Lugo destituyó a los comandantes del Ejército, de la Marina y de la Fuerza Aérea. Lugo denunció que persistían "bolsones retardatarios" entre los uniformados, pero desestimó la posibilidad de un golpe de Estado. Ésta fue la cuarta vez en el año que el Comandante en Jefe efectuó cambios en las cúpulas castrenses.

\section{Poder Legislativo}

El Poder Legislativo es ejercido por el Congreso, compuesto de la Cámara de Senadores y la Cámara de Diputados. Sus miembros son elegidos cada cinco años en comicios simultáneos con los de la presidencia y vicepresidencia de la república, a través de listas "sábanas", o cerradas y bloqueadas. El sistema electoral se basa en la representación proporcional, utilizando el método D'Hondt para asignar escaños. Los 45 senadores son elegidos en una sola circunscripción nacional, lo que facilita la posibilidad de que partidos pequeños puedan ganar escaños. Los 80 diputados son elegidos en circunscripciones departamentales. El Departamento Central elige 19 diputados, pero seis de los 17 departamentos eligen sólo dos diputados, y dos departamentos eligen sólo a uno, reduciendo por mucho la proporcionalidad del voto y favoreciendo a los partidos tradicionales, los partidos Colorado (Asociación Nacional Republicana-Partido Colorado o ANR) y Liberal (Partido Liberal Radical Auténtico o PLRA). Los dos partidos dominan en la Cámara baja con 59, o 73,75\%, de los 80 diputados, y en la Cámara alta con 29 , o $64,44 \%$, de los 45 senadores.

Las leyes electorales del Paraguay requieren elecciones internas para la selección de candidaturas en todos los partidos y movimientos. La nómina final de cada partido está formada también por el sistema D'Hondt, que permite que fracciones internas ganen espacios en la lista cerrada del partido y en la legislatura. Esto ha significado que, desde que se promulgó la Constitución de 1992, en la legislatura las bancadas han funcionado más bien por fracción que por partido. En el Senado hay dos bancadas coloradas y dos liberales, más las bancadas del Partido Unión Nacional de Ciudadanos Éticos (PUNACE), Partido Patria Querida (PPQ) y la "Bancada Unificada" de los tres senadores de partidos de la izquierda, miembros de la Alianza Patriótica para el Cambio (APC) del Presidente Lugo. En Diputados hay tres bancadas coloradas y tres liberales, más las bancadas de PUNACE, PPQ y dos "bancadas" de partidos y movimientos de la APC formados por una diputada cada uno. 
Cuadro 7: Conformación de Congreso, miembros por bancada

\begin{tabular}{lcc}
\hline \multicolumn{1}{c}{ Partido } & Senadores & Diputados \\
\hline ANR-Partido Colorado & & 11 \\
$\quad$ Bancada A & 8 & 9 \\
$\quad$ Bancada B & 6 & 10 \\
$\quad$ Bancada C & $1^{*}$ & \\
PLRA & & 15 \\
$\quad$ Bancada A & 11 & 5 \\
$\quad$ Bancada B & 3 & 9 \\
$\quad$ Bancada C & & 15 \\
PUNACE & 9 & 4 \\
PPQ & 4 & 1 \\
"Unificada" & 3 & 1 \\
Movimiento de Participación Ciudadana & & \\
PDP & & \\
\hline
\end{tabular}

* No es miembro de una bancada.

La mesa directiva de las cámaras se renueva cada año, en julio. Es un proceso de negociación que comienza con meses de anticipación, y puede estar o no basado en acuerdos logrados en años anteriores. La Mesa Directiva de la Cámara de Diputados para 2009-2010 está conformada por representantes de las tres bancadas del PLRA y del PUNACE. El presidente de la Cámara de Diputados es Enrique Salyn Buzarquis Cáceres (PLRA Bancada A), quien fue reelecto por un segundo período en julio de 2009.

En el Senado se realizaron cambios en la Mesa Directiva. Para 2008-2009, el presidente de la Mesa Directiva y por ende Presidente del Congreso era Enrique González Quintana del PUNACE, y la mesa estaba integrada por representantes también del PLRA Bancada A y la Bancada Unificada. Para 2009-2010 fue elegido Miguel Carrizosa Galiano del PPQ, con apoyo de los Colorados y la Bancada Unificada. Es importante destacar que el PPQ, que no apoya al gobierno legislativamente, ha tomado una posición de "oposición leal" y no está de acuerdo ni con los intransigentes ni con los que abogan por el juicio político al Presidente Lugo. Al momento de formar la nueva mesa directiva, el Directorio del PLRA votó por abandonar la APC de Lugo, aunque no necesariamente dejar de apoyar al gobierno.

\section{Poder Judicial}

Como hemos ya visto, la institución que goza de menor confianza es el Poder Judicial, que los paraguayos consideran muy viciado por prácticas corruptas y que no comulga con la defensa del estado de derecho. La Constitución de 1992 pretende darle al Poder Judicial la independencia que nunca conoció durante la dictadura de Alfredo Stroessner (1954-1989). Específicamente, los procesos de nombramiento de magistrados y los ministros de la Corte Suprema pretenden limitar la influencia política, pero ésta es una meta aún no lograda. 
La Corte Suprema de Justicia está integrada por nueve miembros, nominados por el Consejo de la Magistratura. El Consejo presenta una terna de nominados al Senado, que designa al nuevo ministro con el acuerdo del Poder Ejecutivo. En su turno, el Consejo está conformado por ocho miembros, con un representante de la Corte, del Poder Ejecutivo, del Senado, de Diputados, dos profesores de derecho y dos abogados elegidos por todos los abogados matriculados en el país. Para las magistraturas en las jurisdicciones inferiores y los fiscales, el Consejo presenta ternas para que las designe la Corte Suprema.

Al comenzar el año la Corte Suprema tenía sólo ocho miembros, después de la jubilación, requerida por la Constitución, del Ministro Wildo Rienzi al cumplir 75 años de edad. Rienzi está afiliado con el Partido Colorado. El Senado designó a Agustín Lovera Cañete para llenar la vacante, con los votos del ANR, PUNACE y un sector del PLRA, que se supuso fuera un acuerdo para designar ministros por el "cuoteo político", o cuotas acordadas por los partidos políticos. El Presidente Lugo se negó a otorgar su acuerdo con dicho nombramiento el 25 de agosto, denunciando el cuoteo político. Pero todavía no se han superado las cuestiones políticas, dado que el Consejo de la Magistratura, por la forma de su conformación, también refleja intereses políticos y personales. Al terminar el año ya había dos vacantes en la Corte Suprema, con la salida en agosto del Ministro José Altamirano quien no fue confirmado por el Senado en su pretensión de renovación en la Corte, y uno de los tres miembros del Tribunal Superior de Justicia Electoral. No se vaticina cómo se van a llenar las vacantes sin un acuerdo más global y político entre los distintos poderes del Estado y de los partidos políticos.

Como para mostrar la confusión institucional entre los poderes del Estado, el año terminó con la Sala Constitucional de la Corte Suprema anulando el juicio político a Bonifacio Ríos y Carlos Fernández Gadea, ministros removidos de la Corte en 2003. La Sala estuvo conformada no por Ministros de la Corte, dado que estos se recusaron del caso, sino por tres miembros del Tribunal de Cuentas, asignados al caso. Su fallo ordenó que Ríos y Fernández fueran repuestos en sus cargos y que el proceso, ya bien congelado, de designar nuevos ministros fuera suspendido. En fin, el Congreso rechazó el fallo y la Corte Suprema suspendió de sus cargos a las tres camaristas del Tribunal de Cuentas.

\section{TEMAS SALIENTES Y EVALUACIÓN GENERAL}

Un logro singular del gobierno de Fernando Lugo, en el 2009, fue el acuerdo firmado con Luiz Inácio Lula da Silva, Presidente del Brasil el 25 de julio, en el cual Brasil acepta que Paraguay le venda la energía producida por la Hidroeléctrica Itaipú directamente al mercado brasileño, y que Brasil aumente la compensación casi por un factor de tres hasta US\$ 360 millones. Aunque al acuerdo le falta mucho para satisfacer los reclamos de los paraguayos más nacionalistas, Lugo puede contar con haber cumplido una de las promesas centrales de su campaña electoral. Según un informe del Banco Central del Paraguay, las dos binacionales, Itaipú y Yacyretá, ingresaron al país US\$ 670 millones en 2009 (ABC Digital, 2010). 
En los temas sociales el gobierno no ha tenido muchos éxitos. La reforma agraria integral no ha avanzado y la potencialidad de conflictos en el campo sigue como una llaga abierta. Proyectos para mejorar la situación de los pueblos indígenas no han empezado porque el gobierno no ha podido resolver un conflicto sobre la dirección administrativa del Instituto Nacional del Indígena (INDI). Después de haber nombrado a Margarita Mbywangi como la primera indígena presidenta del INDI, se la destituyó en diciembre de 2008. Sólo después del nombramiento de Lidia Acuña como presidenta del instituto finalizó el conflicto.

El secuestro del ganadero Fidel Zavala de su estancia en el Departamento de Concepción, el 15 de octubre, por un comando del autonombrado Ejército del Pueblo Paraguayo (EPP) captó fuertemente la atención pública y mostró las debilidades del Estado. Zavala fue puesto en libertad el 17 de enero de 2010, sin que haya caído ningún miembro del EPP. La presencia mínima del Estado en las zonas rurales y la ineficiencia de las fuerzas de seguridad han dado a la EPP una capacidad de burlar al gobierno, sin presentarse como una amenaza directa al poder.

\section{CONCLUSIONES}

Después de las elecciones históricas de 2008, el primer año completo de un gobierno no Colorado demuestra que el cambio no viene sólo de una renovación en el Poder Ejecutivo. Las debilidades del sistema político paraguayo frustraron las posibilidades de dar camino a soluciones a los profundos problemas sociales y de gobernabilidad. El gobierno de Lugo parece incapaz de mediar las fuerzas que en coalición unieron para ganar el poder pero no compartan una visión de las políticas más indicadas para el país. Al final del año, el gabinete parece algo más coherente pero marcado todavía con conflictos internos.

Los índices económicos y sociales no marcan mejoras importantes para que se pueda esperar progreso en la resolución de las muchas cuestiones pendientes en el país. En fin, las debilidades internas de la coalición que llevó a Fernando Lugo a la presidencia en 2008, los límites de las capacidades reales del Poder Ejecutivo y los grandes desafíos presentados por la estructura socioeconómica de Paraguay y por la herencia política de más de 60 años de gobierno de partido único hicieron de 2009 un año en el cual muy poco ha parecido cambiar.

\section{REFERENCIAS}

ABC Digital. 2009. "Se redujo la concentración de tierras en 17,5\%, de acuerdo al censo 2008", 20 agosto. [En línea] http:/ / www.abc.com.py/2009/08/20/nota/15494-Se-redujo-la-concentración-de-tierrasen-17,5,-de-acuerdo-al-censo-2008/. [Consulta 6-6-2010].

ABC Digital. 2010. "Binacionales ingresaron al país 670 millones de dólares", 3 de febrero. [En línea] http:/ /www.abc.com.py/2010/02/03/nota/72750-Binacionales-ingresaron al-país 670-millonesde-dólares /. [Consulta 6-6-2010]. 
Angus Reid Global Monitor. 2009. "Half of Paraguayans Satisfied with Lugo", 21 August. [En línea] http://www.angus -reid.com/polls/view/34054/half_of_paraguayans_satisfied_with_lugo. [Consulta 30-5-2010].

Banco Central del Paraguay (BCP). 2009. Informe Económico, diciembre. [En línea] http:/ /www.bcp.gov. py/index.php?option=com_content\&task=view\&id=140\&Itemid=364. [Consulta 30-5-2010].

Banco Central del Paraguay (BCP). 2010. Informe Económico, enero. [En línea] http:/ / www.bcp.gov.py/ index.php?option=com_content\&task=view\&id=140\&Itemid=364. [Consulta 30-5-2010].

Banco Mundial. World dataBank. [En línea] http://databank.worldbank.org/ddp/home.do. [Consulta 30-5-2010].

Belmonte, Alejandro. 2009. "Explorando algunos vínculos entre la calidad de la democracia y las élites parlamentarias en América Latina", Universidad de Salamanca, Boletín de Datos de Opinión No 08-09 (junio). [En línea] http://americo.usal.es/oir/Elites/Boletines\%204/8_Belmonte.pdf. [Consulta 30-5-2010].

Brenes, Diego. 2009. "(Des)confianza en los organismos y procesos electorales en América Latina", Universidad de Salamanca, Boletín de Datos de Opinión No 10-09 (julio). [En línea] http:/ /americo. usal.es/oir/Elites/Boletines\%204/10_Brenes.pdf. [Consulta 30-5-2010].

Carrión, Julio F. 2008. "Illiberal Democracy and Normative Democracy: How is Democracy Defined in the Americas?" En Challenges to Democracy in Latin America and the Caribbean: Evidence from the Americas Barometer, 2006-07, Mitchell A. Seligson, ed., Latin American Public Opinion Project, Vanderbilt University, Pp. 21-51. [En línea] http://sitemason.vanderbilt.edu/files/j8Eyre/Challenges_to_ Democracy_in_Latin_America_and_the_Caribbean_Englishv15b_Old_Word_formatted_V8.pdf. [Consulta 30-5-2010].

Corral, Margarita. 2009. "¿Los partidos políticos escuchan a la gente? Opiniones desde las Américas", Perspectivas desde el Barómetro de las Américas ( $\left.\mathrm{N}^{\circ} 12\right)$. [En línea] http:/ / sitemason.vanderbilt.edu/ lapop/AmericasBarometerInsightsSeries. [Consulta 30-5-2010].

Cruz, José Miguel. 2009. “¿Deben las autoridades respetar la ley cuando luchan en contra del crimen?", Perspectivas desde el Barómetro de las Américas ( $\mathrm{N}^{\mathrm{o}}$ 19). [En línea] http://sitemason.vanderbilt.edu/ lapop/AmericasBarometerInsightsSeries. [Consulta 30-5-2010].

Dirección General de Estadística, Encuestas y Censos (DGEEC). 2008a. Encuesta Permanente de Hogares, 2008. [En línea] http:/ /www.dgeec.gov.py/. [Consulta 30-5-2010].

Dirección General de Estadística, Encuestas y Censos (DGEEC). 2008b. “Mejora de la metodología de medición de pobreza en Paraguay Resultados 1997-2008". [En línea] http://www.dgeec.gov.py/. [Consulta 30-5-2010].

Fogel, Ramón, y Marcial Riquelme, compiladores. 2005. Enclave Sojero: Merma de Soberanía y Pobreza. Asunción: Centro de Estudios Rurales Interdisciplinarios.

Montalvo, Daniel. 2009a. "Confianza en los tribunales electorales", Perspectivas desde el Barómetro de las Américas ( $\mathrm{N}^{\mathrm{0}}$ 23). [En línea] http:/ / sitemason.vanderbilt.edu/lapop/AmericasBarometerInsightsSeries. [Consulta 30-5-2010].

Montalvo, Daniel. 2009b. “¿Confía usted en sus fuerza armadas?”, Perspectivas desde el Barómetro de las Américas ( $\mathrm{N}^{\circ}$ 27). [En línea] http:/ / sitemason.vanderbilt.edu/lapop/AmericasBarometerInsightsSeries. [Consulta 30-5-2010].

Orces, Diana. 2009a. "Apoyo popular para imponer límites en los partidos de oposición por el ejecutivo", Perspectivas desde el Barómetro de las Américas 2009 (N 9). [En línea] http:/ / sitemason.vanderbilt.edu/ lapop/AmericasBarometerInsightsSeries. [Consulta 30-5-2010].

Orces, Diana. 2009b. "Apoyo popular a un gobierno sin el Congreso", Perspectivas desde el Barómetro de las Américas 2009 ( $N^{o}$ 25). [En línea] http:// sitemason.vanderbilt.edu/lapop/AmericasBarometerInsightsSeries. [Consulta 30-5-2010].

Brian Turner es profesor y Chair del Departamento de Ciencia Política del Randolph-Macon College, Ashland, Virginia. P.O. Box 5005 Ashland, VA 23005-5505.

bturner@rmc.edu 